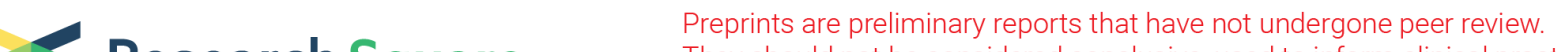 Research Square They should not be considered conclusive, used to inform clinical practice, or referenced by the media as validated information.
}

\section{Altered expression of aggrecan, FAM20B, B3GALT6, and EXTL2 in patients with osteoarthritis and Kashin-Beck disease}

Jian Lei

Xi'an Jiaotong University

Liyun Wang

Xi'an Jiaotong University

Huan Deng

Xi'an Jiaotong University

Yan Ran

Xi'an Jiaotong University

Yizhen LV

Xi'an Jiaotong University

Xiong Guo

Xi'an Jiaotong University

Amhare Abebe Feyissa

Xi'an Jiaotong University

Jing Han ( $\nabla$ bbbishop@126.com )

Xi'an Jiaotong University https://orcid.org/0000-0003-1460-0114

Mikko J Lammi

Xi'an Jiaotong University

\section{Research article}

Keywords: Kashin-Beck disease; Osteoarthritis; Chondroitin sulfate; Immunohistochemistry

Posted Date: September 23rd, 2019

DOl: https://doi.org/10.21203/rs.2.14817/v1

License: (c) (1) This work is licensed under a Creative Commons Attribution 4.0 International License. Read Full License

Version of Record: A version of this preprint was published at CARTILAGE on June 9th, 2020. See the published version at https://doi.org/10.1177/1947603520932199. 


\section{Abstract}

Background: Kashin-Beck disease (KBD) is an endemic osteoarticular disease, which has had a high prevalence in China. Osteoarthritis (OA) is the dominating proportion of osteoarticular disease. The objective of this study is to investigate the expression of enzymes involved in synthesis and modification of chondroitin sulfate (CS) in knee cartilage tissue of osteoarthritis (OA) and Kashin-Beck disease (KBD) patients.

Methods: Knee articular cartilage samples were obtained from 18 age and gender matched donors. Hematoxylin \& eosin staining, toluidine blue staining, and immunehistochemical staining were performed to estimate the expression level and localization of aggrecan and the enzymes associated with CS synthesis and modification (FAM20B, GalT-II, EXTL2). Rank-based analyses of variance (ANOVA) test was used for the multiple comparisons of discrepancy in the positive staining rate among the KBD, OA, and control groups.

Results: Decreased proteoglycans were observed in OA and KBD compared with control group. In line with these expression trends, the positive staining rates of aggrecan were lower in $\mathrm{KBD}$ and $\mathrm{OA}$ group than control group. The positive staining rates of CS chain modifying enzymes FAM2OB, GalT-II, and EXTL2 were also decreased in $\mathrm{OA}$ and $\mathrm{KBD}$ group.

Conclusion: In conclusion, our study uncovered that the enzymes involved in synthesis and modification of CS can influence the expression synthesis of aggrecan and CS. Further investigation of these enzymes can provide new theoretical and experimental targets for OA and KBD pathogenesis studies.

\section{Introduction}

Osteoarticular disease is one of the most prevalent chronic joint diseases in the world [1,2]. Osteoarthritis $(\mathrm{OA})$ is the major proportion of osteoarticular disease and causes joint pain and stiffness [3]. The pathologic changes in $\mathrm{OA}$ include progressive degradation of articular cartilage, formation of osteophytes, variable degrees of inflammation of the synovium, and subchondral bone remodeling $[4,5]$. Unlike OA, Kashin-Beck disease (KBD) is an endemic osteoarticular disease, which has been of high prevalence and morbidity in the Eastern Siberia of Russia, North Korea, and northern-east to southernwest belt in China [6]. KBD characterizes by degeneration and necrosis in the deep layer of hyaline cartilage, dyschondroplasia, inflammation, and metabolic disorders related to cartilage extracellular matrix (ECM), such as chondroitin sulfate (CS) modifying enzymes [6,7-10].

The ECM of cartilage plays an essential role in tissue and organ morphogenesis, and also contributes to the cell structure and function maintenance of articular cartilage [11]. The ECM degeneration has been demonstrated in OA and KBD pathology, particularly in the necrotic areas [10-13]. ECM consists of large aggregating and small leucine-rich proteoglycans (PGs), hyaluronan, glycoproteins, and collagens $[14,15]$. PGs are important bio-macromolecules composed of core protein and glycosaminoglycan (GAG) chains $[14,16,17]$, and participate in ECM deposition, cell differentiation, adhesion and migration [5]. Aggrecan is 
the principal PG present in the ECM of articular cartilage. As a part of aggrecan, CS is the major component of cartilage and provides resistance to compression [17]. The negative groups of the GAGs can adsorb water and act as hydrogel and lubricant in the joints [18]. Besides, altered expression of CS and decreased content of CS has been observed in OA and KBD [10,11,13].

CS sulfation is an essential process for the completion of PG function. A series of glycosyl transferase and sulfonyl transferase are involved in the synthesis and sulfation modification process of CS, including enzymes such as family with sequence similarity 20 , member B (FAM20B, XylK), $₫-1,3-$ galactosyltransferase 6 (GalT-II, B3GALT6), and exostosin-like 2 (EXTL2, EXTL2). Fam20B can phosphorylate the xylose residue in the GAG-protein linkage region of PGs, and regulate the rate of GAG chains synthesis $[16,19,20,21]$. GalT-II is an important enzyme participating in the biosynthesis of GAG tetrasaccharide linkage region [18,22], while EXTL2 functions as a suppressor of Fam20B and induces CS biosynthesis [23].

Therefore, we speculate that the dysfunction of CS synthesis and sulfation modifying enzymes may be involved in the articular cartilage damage in patients with $\mathrm{OA}$ and KBD. However, studies related to these enzymes are still limited, and the mechanisms need to be further investigated. In this study, hematoxylin \& eosin (HE) staining was used to observe the number and morphology of chondrocytes; toluidine blue (TB) staining was used to evaluate the change of matrix and the sulfated GAGs of articular cartilage in different layers of the KBD, OA, and control groups. toluidine blue (TB) staining was performed to investigate the expression levels of three CS metabolic enzymes (FAM20B, GaIT-II, EXTL2) and aggrecan in the articular cartilage of KBD, OA, and control groups.

\section{Materials And Methods}

\section{Sample collection and grouping}

Knee articular cartilage samples were obtained from joint replacement or debridement operation of 18 age and gender matched donors $(\mathrm{KBD} n=6, \mathrm{OA} n=6$, control $n=6)$ [9]. The Chi-square test $\left({ }^{2}\right.$ gender $=$ $0.560)$ and Student's t-test $\left(F_{\text {age }}=2.485\right)$ analyses of the samples showed that subjects in the three groups did not differ significantly $(p>0.05)$ in gender and age distribution. KBD cartilage samples were collected from patients with KBD assessed by clinical classification of grades II/III, and diagnosed according to the diagnostic criteria for Kashin Beck disease (WS/T207-2010) and the X-ray films of the right hand, knee and hip [24]. OA samples were collected from donors living in non-KBD areas of Shanxi

province, who were OA was diagnosed based on the Western Ontario and McMaster Universities OA Index (WOMAC) [25] graded and diagnosed based on histopathology $[8,26]$. The normal control samples were collected from donors living in non-KBD areas of Shanxi province who received total knee arthroplasty or amputations surgery. This investigation was approved by the Human Ethics Committees of Xi'an Jiaotong University. All the donors had provided a written informed consent for the study participation and publication of their individual clinical details and images. 
Once collected, the articular cartilage tissues were fixed with $4 \%(\mathrm{w} / \mathrm{v})$ paraformaldehyde for 24 hours, and decalcified in $10 \%(\mathrm{w} / \mathrm{v})$ ethylene-diamine tetra-acetic acid disodium salt $\left(E D T A-\mathrm{Na}_{2}\right)$ for 4 weeks with regular agitation to eliminate calcium. Then, all samples were embedded in paraffin and cut into serial $6-\mu \mathrm{m}$-thick sections in the coronal direction. The sections were deparaffinized in xylene, followed by decreasing concentrations of ethanol solution (100-80\%). After deparaffinization, the sections were stained with $\mathrm{HE}$ and $0.1 \%(\mathrm{w} / \mathrm{v})$ TB solution. After rinsing with running distilled water for 10 minutes, the sections were dehydrated by increasing concentrations of ethanol solution (80-100\%). Finally, the sections were dehydrated in xylene before covering with coverslips and observation with microscope.

\section{Immunohistochemical staining}

Immunohistochemical stainings were performed to investigate the expression levels of three CS metabolic enzymes and aggrecan in the articular cartilage of KBD, OA, and control groups. The protocols of immunohistochemical stainings were based on the method used in our previous study [9]. Polyclonal rabbit primary antibodies against human FAM20B (ab121261, Abcam, Cambridge, MA, USA), human GalT-II (ab103375, Abcam), human ACAN (13880-1-AP, Proteintech, Rosemont, IL, USA), and monoclonal rabbit primary antibody against human EXTL2 (ab168391, Abcam) diluted (1:50) in PBS were used in immunohistochemical stainings.

\section{Imagecapture}

Representative regions of the sections were photographed using Olympus BX51 microscope (Olympus, Tokyo, Japan). The sections were observed at 100x magnification and six images from superficial, intermediate, and deep layers from each section of the different stainings were captured. The resolution of all images $1200 \times 1600$ pixels, and they were saved in TIFF format.

\section{Statistical analysis}

For HE stainings, the total cell numbers in images were calculated. For TB staining, the rates of staining area were quantificated with Image $\mathrm{J}$ software (NIH, USA) with a threshold of 180 . For IHC staining, the rates of positively stained cells in superficial, medial, and deep layers of FAM20B, EXTL2, and GalT-II (only cells with integrity morphology were counted) from six images per one sample. The positive staining rates were analyzed with SAS 9.3 software. The normality test and homogeneity of variance were conducted for KBD, OA and control group. As a result, not all data satisfied normality and homogeneity of variance. The data from all the 3 groups was calculated as median (P25, P75). Rank-based analyses of variance (ANOVA) test was used for the multiple comparisons of discrepancy in the positive staining rate among the KBD, OA, and control groups. The results are represented by $T$ value. Statistical significance was accepted when $p<0.05$.

\section{Results}


The integrity and continuity of superficial layer in $\mathrm{OA}, \mathrm{KBD}$ groups were interrupted compared with the control group (Figure 1, Table 1). The chondrocyte density in superficial layer decreased and chondrocytes were in a loose arrangement. Unlike OA or control, the chondrocytes in the deep layer of KBD were circular or ovoid and loosely arranged, and some cells appeared to be in clustered aggregates. The numbers of chondrocytes in superficial layer of OA and KBD were lower than in the control one. The chondrocytes in the deep layer were long spindle- or polygon-shaped and in dense arrangement, which suggested that the morphology of chondrocyte had changed and necrosis or apoptosis occurred in the damaged cartilage.

\section{TB staining analysis}

TB staining was performed to investigate the sulfated GAGs of articular cartilage. In the controls, the chondrocyte nuclei had deep staining intensity, and ECM was rather homogenously stained (Figure 2). Compared with the control group, the depth and area of ECM staining was obviously decreased in OA and KBD group (Figure 2, Table 1). In addition, the stained areas had uneven distribution, and some parts of the superficial articular cartilage were stainless. The deficient or uneven staining thus indicated the lower PG and/or GAG sulfation level in OA and KBD groups.

\section{Immunohistochemical staining of aggrecan, FAM20B, EXTL2, and GalT-II}

In IHC staining of agggrecan, positive areas of aggrecan distributed in the ECM around the chondrocytes (Figrue 3). Therefore, we used the positive staining areas to estimate the expression level of aggrecan. The positive staining areas were highest in the middle, then superficial and deep layers in different layers of the KBD, OA, and control groups. Statistical analysis indicated that the positive staining areas decreased significantly in the deep layers of KBD and OA groups compared with control group (Table 1).

The positive staining cells of FAM20B were distributed to the cytoplasm and among the chondrocytes. The positive staining rates of FAM2OB in different layers of KBD, OA, and control group were highest in the middle, then deep and superficial layers (Figure 4). The positive staining rates of FAM20B were diminished in the superficial, middle, and deep layers of OA and KBD group compared with control group (Figure 4, Table 1).

The positive staining cells of GalT-II were distributed to the cytoplasm and among chondrocytes. In the control group, the positive staining rates were highest in the superficial, then middle and deep layers (Figure 5). In OA and KBD groups, loss of positive staining rates were observed in the superficial layer, and in the middle layer of OA group compared with controls (Figure 5, Table 1).

The positive staining cells of EXTL2 was rather abundant in the control group (Figure 6, Table 1) In OA group, the positive staining rates of EXTL2 decreased compared with the control in the middle and deep layers, while in KBD group, the positive staining rates of EXTL2 decreased in all layers compared to the control group (Figure 6, Table 1). 


\section{Discussion}

The results of our study demonstrated the decreased expression of FAM20B, GalT-II and the increased expression of EXTL2 in in OA and KBD, which involved in CS synthesis and modification processes. Besides, the expression of aggrecan were lower in the deep layer of KBD and OA group compared with control. Considering the function of the enzymes, we speculate that the altered expression of these enzymes may explain the decreased expression of aggrecan.

Aggrecan is the most crucial for the proper functioning of articular cartilage and chondrocytes [17,27], and it functions as lubricant in the biomechanical properties of cartilage $[11,28]$. Aggrecan can create a high osmotic activity and maintain collagen fiber assembly to create swelling pressure for the load bearing capability of the cartilage $[29,30]$. Our results indicate that the immunostaining for aggrecan are lower in the deep layer of KBD and OA group compared with control. Besides, TB staining also showed that loss of staining occurred in KBD and OA. CS is an important part of aggrecan, and the decreased of aggrecan can reflect the defective function of CS.

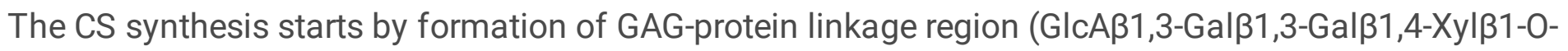
Ser). The elongation of these GAG chains on the core protein occurs then by stepwise addition of individual sugars residues by a series of glycosyl transferase and sulfonyl transferases [18,31]. The first step in the tetrasaccharide linker formation is the xylosylation of the core protein on specific serine residues by xylosyltransferase $[19,22]$. Fam20B is a xylose kinase, which can phosphorylate the xylose residue of the tetrasaccharide linker. Fam20B is an essential switch for the regulation of the PGs-GAGs formation and enhancing the synthesis of CS $[16,19,32]$. Animal experiments have also demonstrated that GAGs in the Fam20B-deficient cartilage were remarkably reduced [21,33]. In our results, the expression levels of FAM20B were lower in all three layers of KBD and OA groups compared with control group. These expression levels were also consistent with the TB results, suggesting that the decreased level of FAM20B may have affected the CS synthesis level.

The second step is the addition of two galactoses catalyzed by two distinct enzymes $\beta 1,4-$ galactosyltransferase 7 (GalT-I) and $\beta 1,3$-galactosyltransferase 6 (GalT-II) [19]. GalT-II is an essential enzyme involved in the biosynthesis of the tetrasaccharide linkage region [22]. Previous study demonstrated that the activity of GalT-II was dramatically increased by Fam20B-dependent xylose phosphorylation, and that the knowdown of Fam20B could suppress GalT-II expression and reduce the rate of GAG synthesis [32,34]. GalT-II can enhance the CS extension on the tetrasaccharide linker [22]. Our results indicate that the expression levels of GalT-II were declined in the superficial and the deep layers of $\mathrm{OA}$ and $\mathrm{KBD}$ compared with control group. This finding is consistent with the TB results, suggesting that a lower expresion level of GalT-II in the diseased cartilage may caused decreased GAGs.

Previous investigations have demonstrated that EXTL2 acts as an inhibitor of CS synthesis by adding Nacetylglucosamine to the end of FAM20B-phosphorylated tetrasaccharide [35,36], which means that extension of CS chain cannot be initiated by EXTL2. Moreover, the phosphorylated pentasaccharide produced by EXTL2 could terminates GAG elongation [36]. Animal experiments have indicated that 
EXTL2 knockout mice produce more GAGs compared to wild type mice [17,37]. The staining for EXTL2 was diminished in the middle and deep layers of OA and KBD compared with control group. Besides, the expression level in superficial layer of KBD decreased significantly compared with OA and control group.

There are still certain limitations in this study due to the procedures used for IHC stainings, which can influence the results, such as specificity of antibody, tissue specimen fixation, antigen retrieval and $\mathrm{pH}$ of repair liquid. Limited number of the samples (in this research $n=18$ ) may lead to some deviations in the results, thus, an experiment with larger sample size would be warranted to validate the results of the experiment.

In summary, our study demonstrated the decreased expression of FAM20B, GalT-II and the increased expression of EXTL2 in OA and KBD. Besides, the expression of aggrecan were lower in the deep layer of $\mathrm{KBD}$ and $\mathrm{OA}$ group compared with control. These enzymes can regulate the modification processes, thus can influence the expression level of aggrecan and CS synthesis. Further investigation of these enzymes can provide new theoretical and experimental targets for $\mathrm{OA}$ and KBD pathogenesis studies. At the same time, targeted therapies could be brought up to the prevention and treatment of OA and KBD.

\section{Abbreviations}

ANOVA: Analyses of variance; CS: Chondroitin sulfate; ECM: Extracellular matrix; EDTA-Na 2 : Ethylenediamine tetra-acetic acid disodium salt; GAG: Glycosaminoglycan; GalT-I: $\beta 1,4$-galactosyltransferase 7; GalT-II: $\beta 1$,3-galactosyltransferase 6; HE: Hematoxylin \& eosin; KBD: Kashin-beck disease; OA: Osteoarthritis; OARSI: Osteoarthritis research society international; PGs: Proteoglycans; TB: Toluidine blue; WOMAC: Western ontario and mcMaster universities OA index

\section{Declarations}

\section{Acknowledgment}

We thank Dr. Weizhuo Wang from the Second Affiliated Hospital of Xian Jiaotong University, Dr. Chao Xu from Linyou County Hospital and all the staff from Linyou County Endemic Disease Control Center for their support and cooperation in the collection of cartilage specimens.

\section{Author contributions}

All authors participated in drafting the article and critically modifying the important content of knowledge, with all authors endorsing the final version. Lei, Wang, Ran had full access to all the data in the study and takes responsibility for the integrity of the data and accuracy of the data analysis. Study conception and design: Han. Acquisition of data: Lei, Wang, Ran, Feyissa, Lv. Analysis and interpretation of data: Lei, Deng, Lammi, Wang. Drafting the article or revising it critically for important intellectual content: Lei, 
Wang, Deng, Ran, Feyissa, Lv, Lammi, Han. Final approval of the version of the article to be published: Lei, Wang, Deng, Ran, Lv, Guo, Feyissa, Han, Lammi

\section{Funding}

This study was supported by Shenzhen Science and Technology Project (JCYJ20170816100755047) and National Natural Science Foundation of China $(81402639,81872567)$. The study sponsors did not involve in the study design, data collection, analysis or interpretation, or in the writing of the manuscript, neither did they affect the decision to submit the manuscript for publication.

\section{Availability of data and materials}

The datasets used and/or analyzed during the current study are available from the corresponding author on reasonable request.

\section{Ethics approval and consent to participate}

This investigation was approved by the Human Ethics Committees of Xi'an Jiaotong University. All the donors had provided a written informed consent for the study participation and publication of their individual clinical details and images.

\section{Consent for publication}

Written informed consent for publication was obtained from the patients included in this study.

\section{Competing interests}

The authors declare no conflicts of interest. The authors have sole responsibility for the writing and content of the paper.

\section{Author details}

${ }^{1}$ Key Laboratory of Environment and Genes Related to Diseases, Xi'an Jiaotong University, Ministry of Education of China, Xi'an, Shaanxi, 710061, PR China. ${ }^{2}$ Shenzhen Institute, Xi'an Jiaotong University, Shenzhen, Guangzhou, 518057, PR China. ${ }^{3}$ Department of Gastroenterology, the First Affiliated Hospital, Health Science Center of Xi'an Jiaotong University, PR China. ${ }^{4}$ Department of Integrative Medical Biology, Umeå University, Umeå 90187, Sweden

\section{Reference}


[1]Roberts SB, Wootton E, De Ferrari L, Albagha OM \& Salter DME. pigenetics of osteoarticular diseases: recent developments. Rheumatology International. 2015;5:1293-1305. doi:10.1007/s00296-015-3260-y

[2] Bijlsma JW, Berenbaum F \& Lafeber F P. Osteoarthritis: an update with relevance for clinical practice. Lancet. 2011;377:2115-2126. doi:10.1016/ S0140-6736(11)60243-2

[3]McAlindon TE, Bannuru RR, Sullivan MC, Arden NK, Berenbaum F, Bierma-Zeinstra SM, Hawker GA, Henrotin Y, Hunter DJ, Kawaguchi H. OARSI guidelines for the non-surgical management of knee osteoarthritis. Osteoarthritis and Cartilage. 2014;22:363-388. doi:10.1016/j.joca.2014.01.003

[4]Loeser RF, Goldring SR, Scanzello CR. \& Goldring MB. Osteoarthritis: a disease of the joint as an organ. Arthritis and Rheumatism. 2012;64:1697-1707. doi:10.1002/art.34453

[5]lozzo RV \& Schaefer L. Proteoglycan form and function: a comprehensive nomenclature of proteoglycans. Matrix Biology. 2015;42:11-55. doi:10.1016/ j.matbio.2015.02.003

[6]Guo X, Ma W, Zhang F, Ren F, Qu C, Lammi MJ. Recent advances in the research of an endemic osteochondropathy in China: Kashin-Beck disease. Osteoarthritis and Cartilage. 2014;22:1774-1783. doi:10.1016/j.joca.2014.07.023

[7]Luo M, Chen J, Li S, Sun H, Zhang Z, Fu Q, Li J, Wang J, Hughes CE, Caterson B, Cao J. Changes in the metabolism of chondroitin sulfate glycosaminoglycans in articular cartilage from patients with KashinBeck disease. Osteoarthritis and Cartilage. 2014;22:986-995. doi:10.1016/j.joca.2014.05.012

[8]Li Y, Zhou Z, Shen B, Yang J, Kang P, Yang X, Liu G, Pei F. Clinical features of Kashin-Beck disease in adults younger than 50 years of age during a low incidence period: severe elbow and knee lesions. Clinical Rheumatology. 2013;32:317-324. doi:10.1007/s10067-012-2115-0

[9]Han J, Li D, Qu C, Wang D, Wang L, Guo X, Lammi MJ. Altered expression of chondroitin sulfate structure modifying sulfotransferases in the articular cartilage from adult osteoarthritis and Kashin-Beck disease. Osteoarthritis and Cartilage. 2017;25:1372-1375. doi:10.1016/j.joca.2017.02.803

[10]Han J, Wang W, Qu C, Liu R, Li W, Gao Z, Guo X. Role of inflammation in the process of clinical Kashin-Beck disease: latest findings and interpretations. Inflammation Research. 2015;64:853-860. doi:10.1007/s00011-015-0861-6

[11] Li S, Cao J, Caterson B \& Hughes C E. Proteoglycan metabolism, cell death and Kashin-Beck disease. Glycoconjugate Journal. 2012;29:241-248. doi:10.1007/ s10719-012-9421-2

[12]Duan C, Guo X, Zhang XD, Yu HJ, Yan H, Gao Y, Ma MJ, Gao ZQ, Xu P, Lammi MJ. Comparative analysis of gene expression profiles between primary knee osteoarthritis and an osteoarthritis endemic to Northwestern China, Kashin-Beck disease. Arthritis Rheumatism. 2010;62:771-780. doi:10.1002/ art.27282 
[13]Gualeni B, Facchini M, De Leonardis F, Tenni R, Cetta G, Viola M, Passi A, Superti-Furga A, Forlino A, Rossi A. Defective proteoglycan sulfation of the growth plate zones causes reduced chondrocyte proliferation via an altered Indian hedgehog signalling. Matrix Biology. 2010;29:453-460. doi:10.1016/ j.matbio.2010.05.001

[14] Vynios DH. Metabolism of cartilage proteoglycans in health and disease. BioMed Research International. 2014;2014:452315. doi:10.1155/2014/452315

[15]Responte DJ, Natoli RM \& Athanasiou KA. Collagens of articular cartilage: structure, function, and importance in tissue engineering. Critical Reviews in Biomedical Engineering. 2007;35:363-411. doi:10.1615/CritRevBiomedEng. v35.i5.20

[16] Shaukat I. Investigation of the role of xylosyltransferases I and II and of the kinase Fam20B in the regulation of proteoglycan synthesis, Université de Lorraine. 2015

[17] Kiani C, Chen L, Wu y, Yee A \& Yang BB. Structure and function of aggrecan. Cell Research. 2002;12:19-32. doi:10.1036/sj.cr.7290106

[18]Li L, Ly M \& Linhardt RJ. Proteoglycan sequence. Molecular BioSystems. 2012;1613-1625. doi:10.1039/c2mb25021g.

[19] Koike T, Izumikawa T, Tamura JI \& Kitagawa H. FAM20B is a kinase that phosphorylates xylose in the glycosaminoglycan-protein linkage region. Biochemical Journal. 2009;421:157-162. doi:10.1042/BJ20090474

[20] Koike T, Izumikawa T, Sato B \& Kitagawa H. Identification of phosphatase that dephosphorylates xylose in the glycosaminoglycan-protein linkage region of proteoglycans. Journal of Biological Chemistry. 2014;289:6695-6708. doi:10.10 74/jbc.M113.520536

[21]Ma P, Yan W, Tian Y, Wang J, Feng J, Qin C, Cheng Y, Wang X. Inactivation of Fam20B in joint cartilage leads to chondrosarcoma and postnatal ossification defects. Scientific Reports. 2016; 6:29814. doi:10.1038/srep29814

[22]Nakajima M, Mizumoto S, Miyake N, Kogawa R, lida A, Ito G, Kitoh H, Hirayama A, Mitsubuchi $H$, Miyazaki O. Mutations in B3GALT6, which encodes a glycosaminoglycan linker region enzyme, cause a spectrum of skeletal and connective tissue disorders. American Journal of Human Genetics.

2013;92:927-934. doi:10.1016/j.ajhg.2013.04.003

[23]Mikami T \& Kitagawa H. Biosynthesis and function of chondroitin sulfate. Biochimica et Biophysica Acta. 2013;1830:4719-4733. doi:10.1016/ j.bbagen.2013.06.006

[24]China. Ministry of Health of the People's Republic of China. Ministry of Health of the People's Republic of China. Vol. WS/T207- 2010 (Beijing, 2010). 
[25]Preedy VR \& Watson RR. Western Ontario and McMaster universities osteoarthritis index. (Springer, 2010).

[26]Pritzker KP, Gay S, Jimenez SA, Ostergaard K, Pelletier JP, Revell PA, Salter D, van den Berg WB. Osteoarthritis cartilage histopathology: grading and staging. Osteoarthritis and Cartilage. 2006;14:13-29. doi:10.1016/j.joca.2005.07.014

[27]Ingavle GC, Frei AW, Gehrke SH \& Detamore MS. Incorporation of aggrecan in interpenetrating network hydrogels to improve cellular performance for cartilage tissue engineering. Tissue Engineering. 2013;19:1349-1359. doi:10.1089/ ten.TEA.2012.0160

[28] Nia HT, Ortiz C, Grodzinsky A. Aggrecan: approaches to study biophysical and biomechanical properties. Methods in Molecular Biology. 2015;1229:221-237. doi:10.1007/978-1-4939-1714-3_20

[29]Morawski M, Brückner G, Arendt T \& Matthews RT. Aggrecan: Beyond cartilage and into the brain. International Journal of Biochemistry and Cell Biology. 2012; 44:690-693.

doi:10.1016/j.biocel.2012.01.010

[30]Stattin E, Wiklund F, Lindblom K, Onnerfjord P, Jonsson Bjornanders, Tegner Yelverton, Sasaki Takako, Struglics A, Lohmander Stefan, Dahl Niklas. A missense mutation in the aggrecan C-type lectin domain disrupts extracellular matrix interactions and causes dominant familial osteochondritis dissecans. American Journal of Human Genetics. 2010;86:126-137. doi:10.1016/ j.ajhg.2009.12.018

[31]Kitagawa H, Tsutsumi K, Ikegamikuzuhara A, Nadanaka S, Goto F, Ogawa T, Sugahara K. Sulfation of the galactose residues in the glycosaminoglycan-protein linkage region by recombinant human chondroitin 6-0-sulfotransferase-1. Journal of Biological Chemistry. 2008;283:27438-27443. doi:10.1074/ jbc.M803279200

[32]Wen J, Xiao J, Rahdar M, Choudhury B, Cui J, Taylor GS, Esko JD, Dixon JE. Xylose phosphorylation functions as a molecular switch to regulate proteoglycan biosynthesis. Proceedings of the National Academy of Sciences USA. 2014;111:15723-15728. doi:10.1073/pnas.1417993111

[33]Eames BF, Yan Y, Swartz ME, Levic DS, Knapik EW, Postlethwait JH, Kimmel. CBMutations in fam20b and xylt 1 reveal that cartilage matrix controls timing of endochondral ossification by inhibiting chondrocyte maturation. PLoS Genetics. 2011;7:e1002246. doi:10.1371/journal.pgen.1002246

[34] Mizumoto S, Yamada S \& Sugahara K. Mutations in biosynthetic enzymes for the protein linker region of chondroitin/dermatan/heparan sulfate cause skeletal and skin dysplasias. Biomedical Research International. 2015;2015:861752. doi:10.1155/2015/861752

[35]Katta K, Imran T, Busse-Wicher M, Gronning M, Czajkowski S, Kusche-Gullberg M. Reduced expression of EXTL2, a member of the exostosin (EXT) family of glycosyltransferases, in human embryonic kidney 
293 cells results in longer heparan sulfate chains. Journal of Biological Chemistry. 2015;290:1316813177. doi:10.1074/jbc.M114.631754

[36]Nadanaka S, Zhou S, Kagiyama S, Shoji N, Sugahara K, Sugihara K, Asano M, Kitagawa H. EXTL2, a member of the EXT family of tumor suppressors, controls glycosaminoglycan biosynthesis in a xylose kinase-dependent manner. Journal of Biological Chemistry. 2013;288:9321-9333. doi:10.1074/ jbc.M112.416909

[37]Nadanaka S \& Kitagawa H. EXTL2 controls liver regeneration and aortic calcification through xylose kinase-dependent regulation of glycosaminoglycan biosynthesis. Matrix Biology. 2014;35:18-24. doi:10.1016/j.matbio.2013.10.010

\section{Tables}

Table 1 Cell number for HE staining, rates of intensive staining area for TB staining, aggrecan and positive staining rates and multiple comparisons for FAM20B, EXTL2 and B3GALT6 from normal, OA and KBD groups. The results are presented as median (P25, P75).

\begin{tabular}{|c|c|c|c|c|c|c|c|}
\hline \multirow[t]{2}{*}{ Enzymes } & \multirow[t]{2}{*}{ Zones } & \multicolumn{3}{|c|}{ Groups } & \multicolumn{3}{|c|}{$|\mathrm{T}|$ value for multiple comparison } \\
\hline & & Control & $\mathrm{OA}$ & KBD & Control-OA & OA-KBD & Control-KBD \\
\hline \multirow[t]{3}{*}{$\mathrm{HE}$} & Superficial & $106.0(64.0,117.0)$ & $38.0(32.0,50.0)$ & $82.0(66.0,92.0)$ & $31.719 * * *$ & $25.62 * * *$ & 6.099 \\
\hline & Middle & $35.0(32.0,50.0)$ & $30.0(27.0,34.0)$ & $40.0(32.0,44.0)$ & $18.794^{* * *}$ & $19.895^{* * *}$ & 1.131 \\
\hline & Deep & $34.0(30.0,38.0)$ & $36.0(32.0,44.0)$ & $37.0(28.0,42.5)$ & 8.102 & 4.78 & 3.321 \\
\hline \multirow[t]{3}{*}{ TB } & Superficial & $62.2(55.0,68.1)$ & $15.9(12.1,23.7)$ & $22.6(1.0,73.3)$ & $17.9^{* * *}$ & $12.2^{* *}$ & $30.1 * * *$ \\
\hline & Middle & $99.9(99.9,99.9)$ & $48.8(37.8,64.6)$ & $42.0(20.0,67.8)$ & $22.794^{* * *}$ & 3.777 & $26.571^{* * *}$ \\
\hline & Deep & $99.7(99.0,100.0)$ & $73.6(54.3,86.3)$ & $76.3(68.4,89.8)$ & $27.167^{* * *}$ & 4.078 & $23.088^{* * *}$ \\
\hline \multirow[t]{3}{*}{ ACAN } & Superficial & $49.41(40.56,64.83)$ & $47.82(17.42,77.88)$ & $60.65(51.44,70.07)$ & 0.3 & 7.527 & 7.227 \\
\hline & Middle & $60.32(53.73,77.06)$ & $80.92(55.79,88.29)$ & $67.76(61.78,72.87)$ & 8.583 & 2.938 & 5.646 \\
\hline & Deep & $29.05(19.89,31.06)$ & $8.87(7.12,12.19)$ & $11.96(11.77,21.47)$ & $13.389 * * *$ & 4.667 & $8.722^{* * *}$ \\
\hline \multirow[t]{3}{*}{ FAM20B } & Superficial & $23.5(18.0,32.0)$ & $5.5(2.0,10.0)$ & $7.0(3.0,29.0)$ & $21.51^{* * *}$ & 8.633 & $12.877^{* * *}$ \\
\hline & Middle & $62.0(40.0,81.0)$ & $35.0(21.0,51.0)$ & $32.0(19.0,55.0)$ & $22.692^{* * *}$ & 1.232 & $23.924^{* * *}$ \\
\hline & Deep & $45.0(27.5,55.5)$ & $12.0(4.0,27.0)$ & $14.5(9.0,63.0)$ & $21.95^{* * *}$ & 10.488 & $11.462^{* * *}$ \\
\hline \multirow[t]{3}{*}{ GalT-II } & Superficial & $89.47(83.33,95.24)$ & $31.0(14.0,47.0)$ & $32.0(16.0,70.0)$ & $46.964^{* * *}$ & 6.88 & $40.084^{* * *}$ \\
\hline & Middle & $67.0(25.0,84.0)$ & $29.0(16.0,74.0)$ & $65.0(38.0,80.0)$ & $21.371^{* * *}$ & $20.498 * * *$ & 0.871 \\
\hline & Deep & $34.0(24.0,66.0)$ & $21.0(16.0,37.0)$ & $27.0(19.0,47.0)$ & $21.701 * * *$ & 8.625 & $21.701^{* * *}$ \\
\hline \multirow[t]{3}{*}{ EXTL2 } & Superficial & $46.6(39.3,58.7)$ & $47.1(33.7,66.2)$ & $5.4(2.6,10.0)$ & 0.138 & $32.313^{* * *}$ & $32.175^{* * *}$ \\
\hline & Middle & $58.0(27.0,83.0)$ & $13.0(6.0,52.0)$ & $25.0(12.0,53.0)$ & $21.381 * * *$ & 8.833 & $12.548^{* * *}$ \\
\hline & Deep & $43.0(36.0,61.0)$ & $11.0(6.0,20.0)$ & $13.0(6.0,20.0)$ & $19.605^{* * *}$ & 0.848 & $20.453^{* * *}$ \\
\hline
\end{tabular}

${ }^{*} p$ value $<0.05 ;{ }^{* *} p$ value $<0.001 ; * * * p$ value $<0.0001$, the bigger the $|\mathrm{T}|$ value, the smaller the $p$ value with statistical significance 
Control
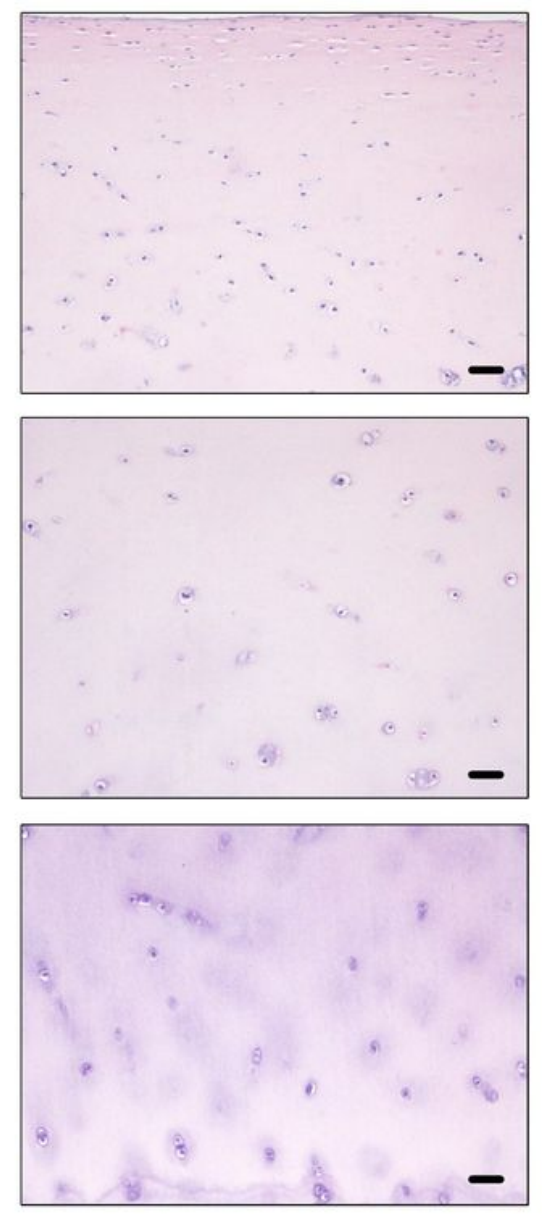

OA
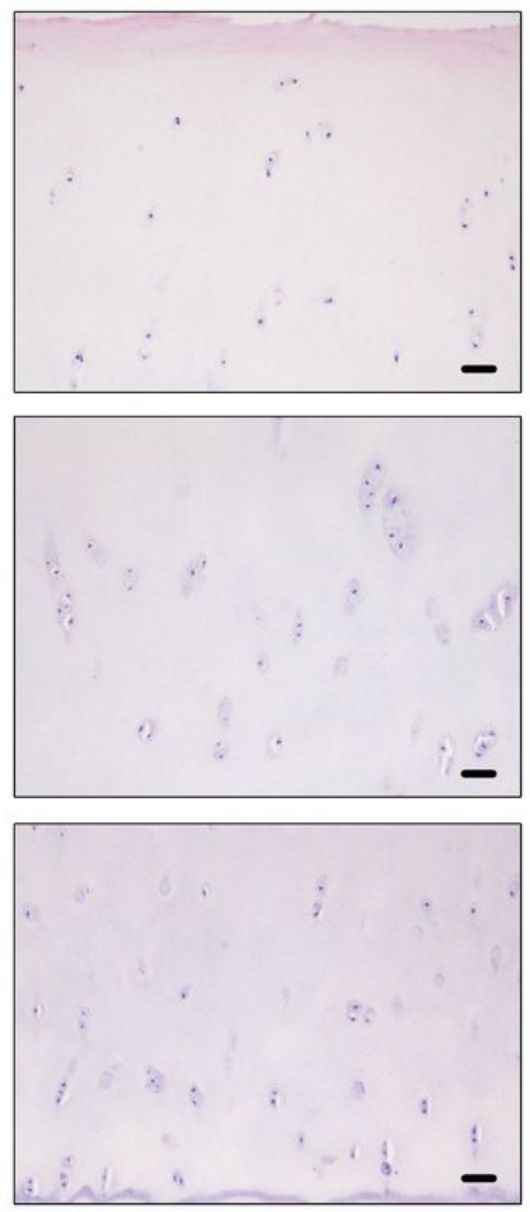

KBD

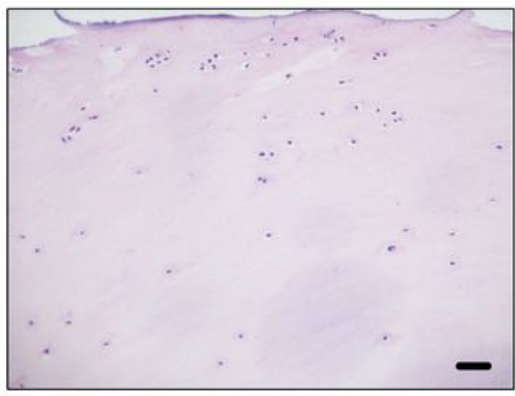

Superficial zone

Middle

zone

Deep

zone

\section{Figure 1}

$\mathrm{HE}$ staining of articular cartilage in control, KBD, and OA groups. In KBD and OA group the cell numbers were much lower than those in control groups in all three layers compared with the control group. Scale bar: $20 \mu \mathrm{m}$. 
Control
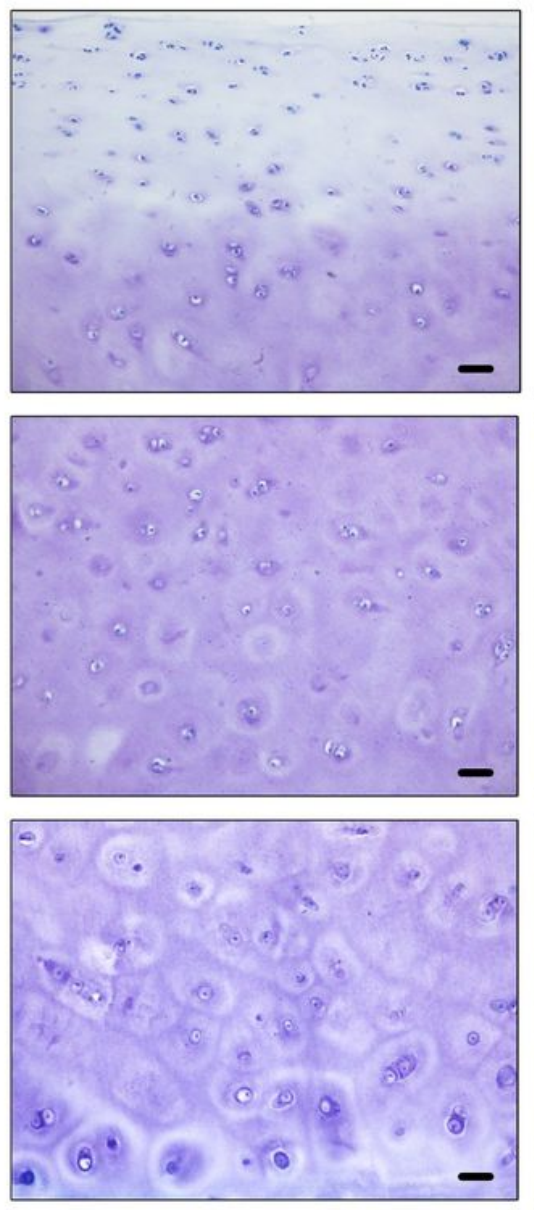

$\mathrm{OA}$
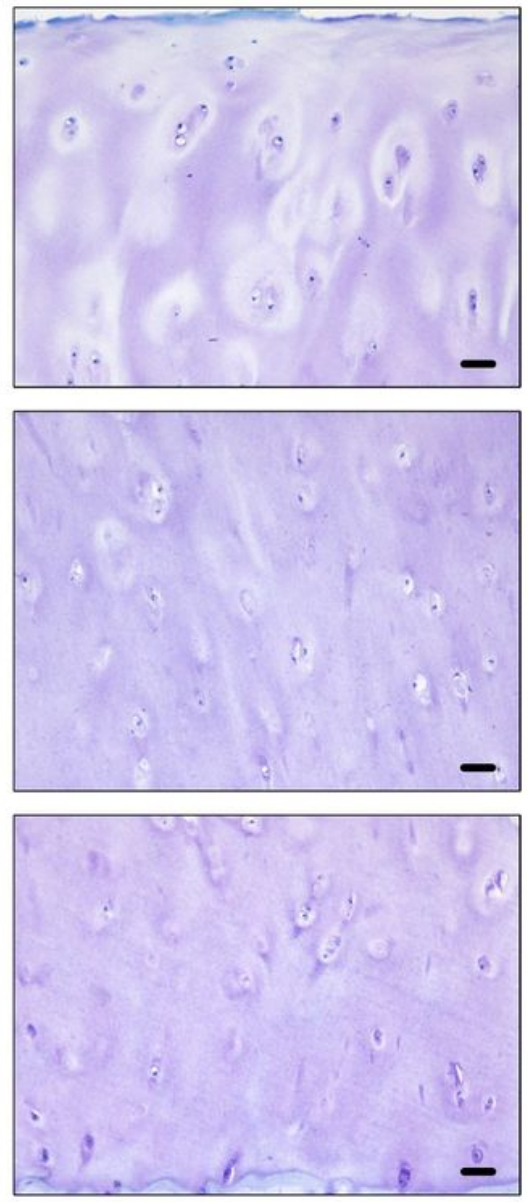

$\mathrm{KBD}$

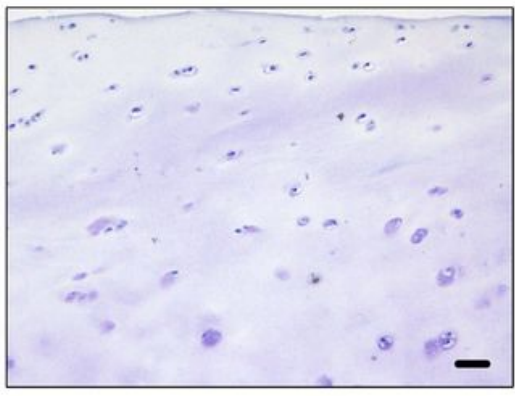

Superficial zone

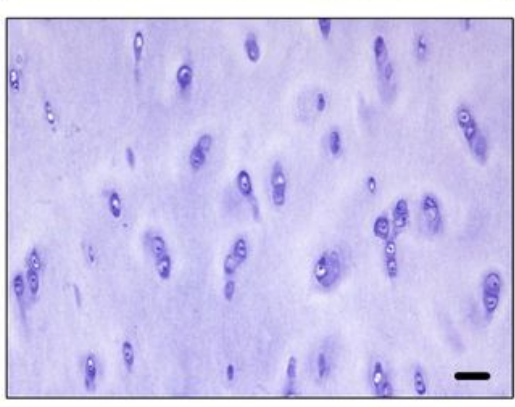

Middle

zone

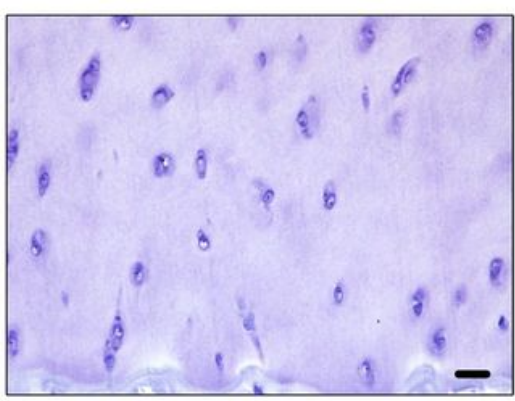

Deep zone

\section{Figure 2}

TB staining of articular cartilage in control, KBD, and OA groups. In KBD and OA group, the average rate of intensive staining area was much lower in all three layers compared with control group. Scale bar: $20 \mu \mathrm{m}$. 
Control
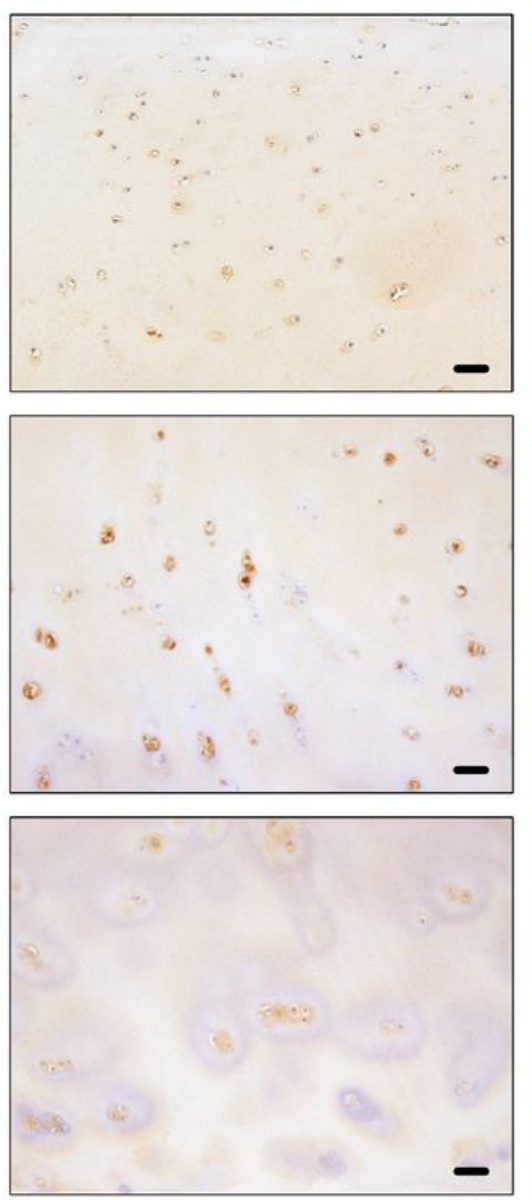

$\mathrm{OA}$
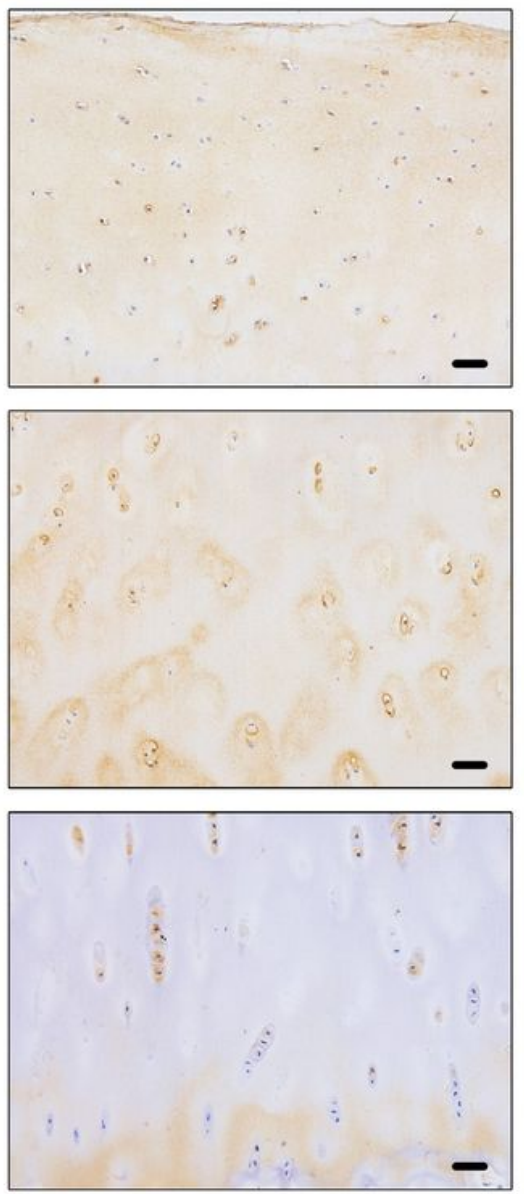

KBD

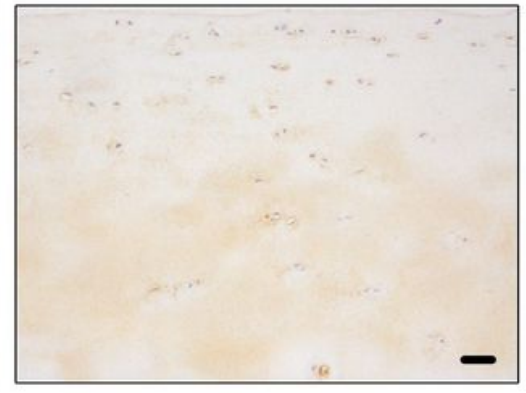

Superficial

zone

Middle

zone

Deep

zone

\section{Figure 3}

IHC staining of aggrecan in the articular cartilage of control, KBD, and OA groups. In deep layers of KBD and $O A$ group, the average rates of intensive staining area were much lower than control group. Scale bar: $20 \mu \mathrm{m}$. 
Control
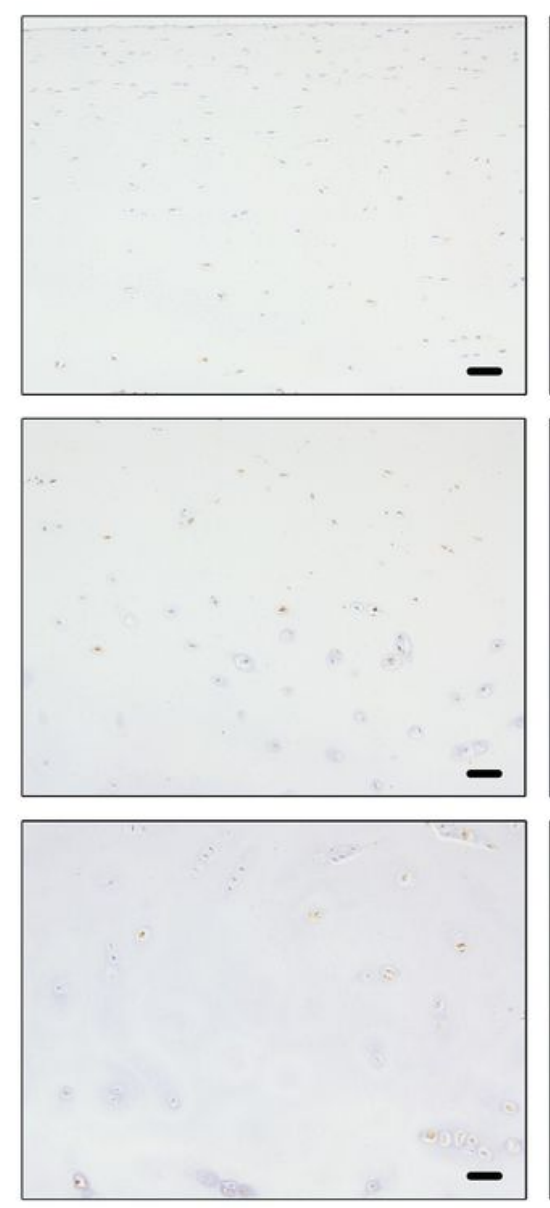

$\mathrm{OA}$
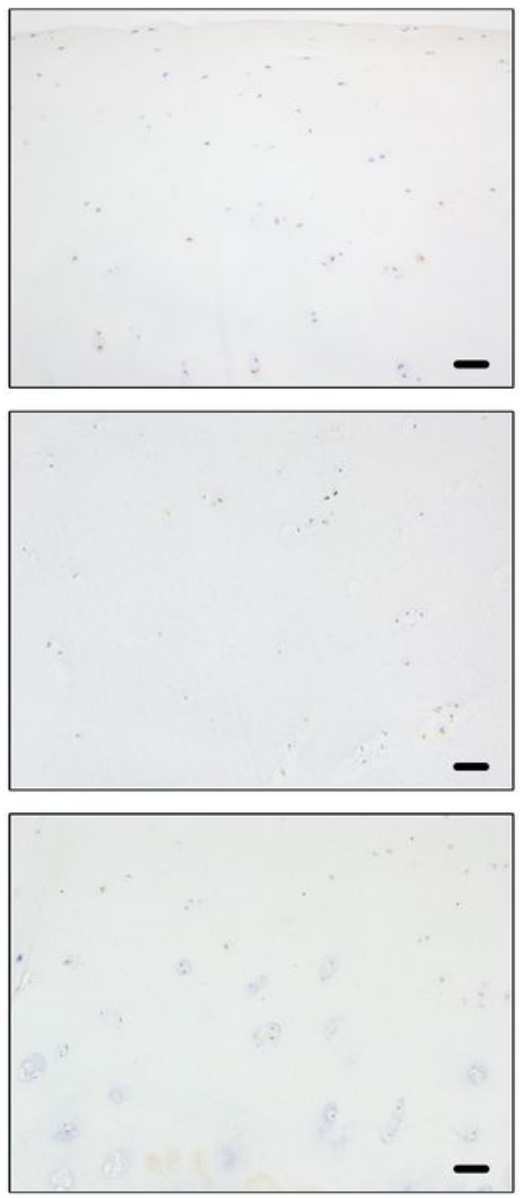

KBD

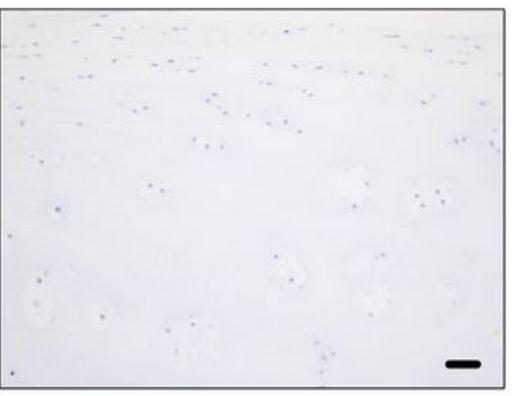

Superficial zone

Middle

zone

Deep

zone

\section{Figure 4}

IHC staining of FAM2OB in the articular cartilage of control, KBD, and OA groups. In all the layers of OA and $\mathrm{KBD}$ group, the positive staining rate in $\mathrm{KBD}$ and $\mathrm{OA}$ group, the positive staining rates were significantly lower compared with control group. Scale bar: $20 \mu \mathrm{m}$. 
Control
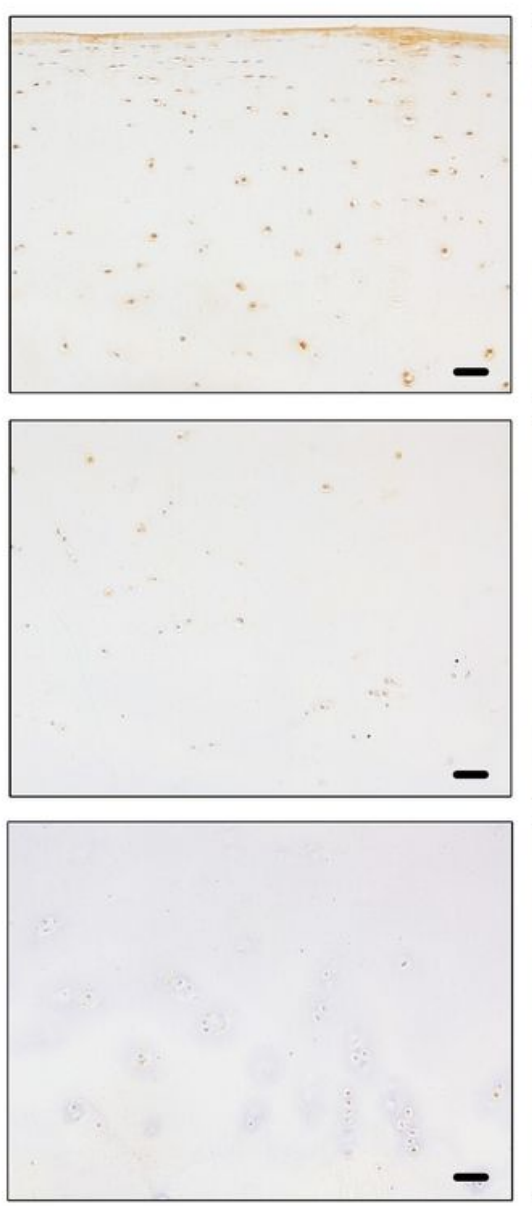

OA
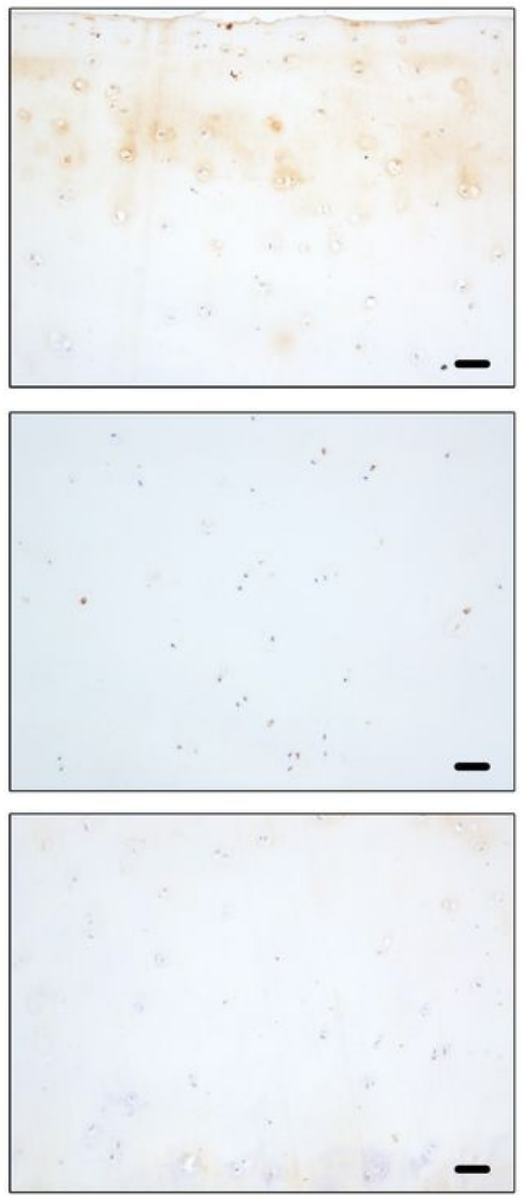

KBD

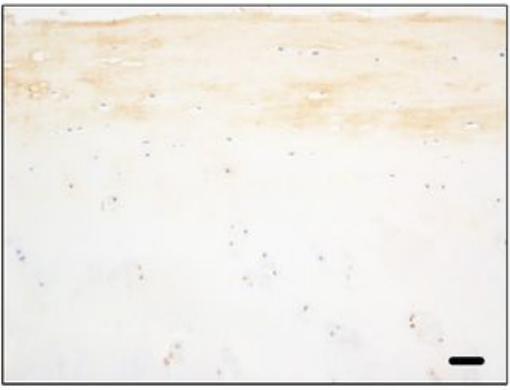

\section{Superficial} zone

Middle zone

\section{Deep} zone

\section{Figure 5}

IHC staining of GalT-II in the articular cartilage of control, KBD, and OA groups. The positive staining rates in superficial and deep layers of KBD and OA groups were markedly lower than control group, and also the middle layer of OA group. While the middle layer of KBD group is higher than OA group. Scale bar: 20 $\mu \mathrm{m}$. 
Control
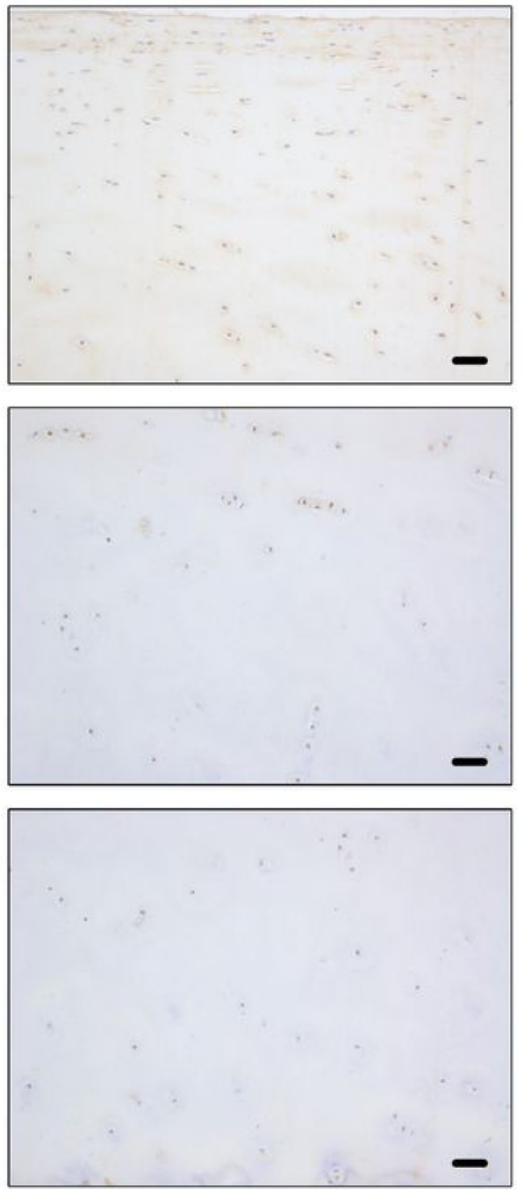

OA
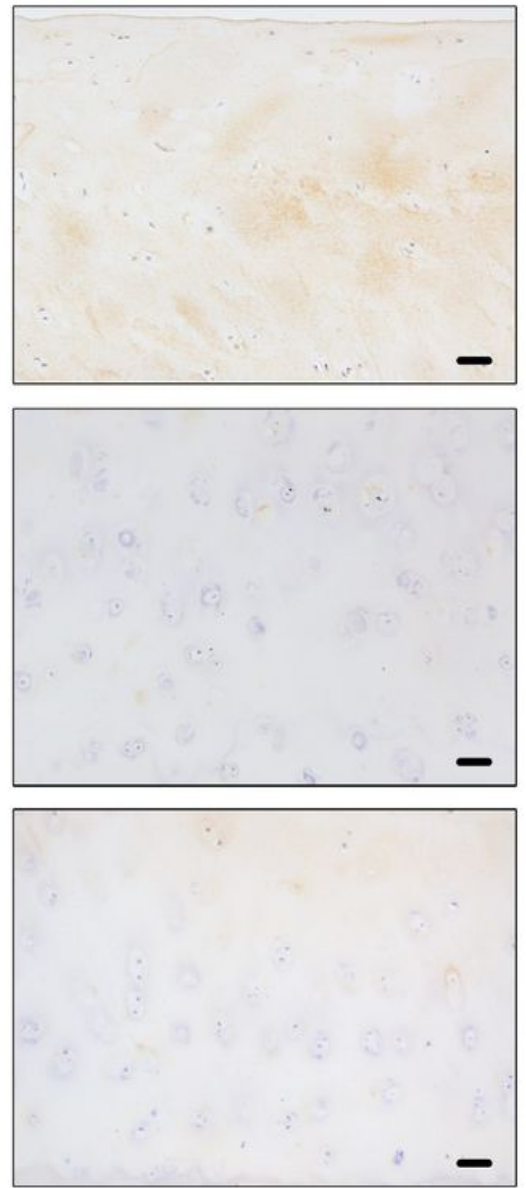

KBD

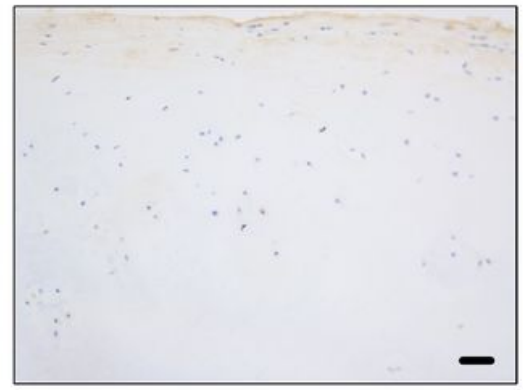

Superficial

zone

Middle zone

Deep zone

\section{Figure 6}

IHC staining of EXTL2 in the articular cartilage of control, KBD, and OA groups. The EXTL2 positive staining rates in the middle and deep layer of KBD and OA groups were lower than control group. and the positive staining rate in OA and control group were higher than KBD group. Scale bar: $20 \mu \mathrm{m}$. 